75

Ournal of

Mechanics of

Materials and Structures
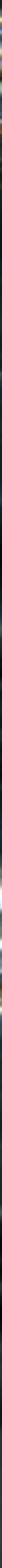


\title{
ORIGIN AND EFFECT OF NONLOCALITY IN A COMPOSITE
}

\author{
STEWART A. SiLLing
}

\begin{abstract}
A simple demonstration of nonlocality in a heterogeneous material is presented. By analysis of the microscale deformation of a two-component layered medium, it is shown that nonlocal interactions necessarily appear in a homogenized model of the system. Explicit expressions for the nonlocal forces are determined. The way these nonlocal forces appear in various nonlocal elasticity theories is derived. The length scales that emerge involve the constituent material properties as well as their geometrical dimensions. A peridynamic material model for the smoothed displacement field is derived. It is demonstrated by comparison with experimental data that the incorporation of nonlocality in modeling improves the prediction of the stress concentration in an open-hole tension test on a composite plate.
\end{abstract}

\section{Introduction}

In typical engineering analysis, the elastic response of a heterogeneous material is treated by applying the classical solid mechanics equations with smoothed (often called "homogenized" or "effective") material properties. These properties can be thought of as those that would be measured using a laboratory test specimen much larger than any internal length scale in the material. For example, we perform an unconfined compression test on a sample of concrete about $15 \mathrm{~cm}$ in diameter and measure the total force as a function of displacement at the ends. We divide the relative displacement at the ends by the specimen length and call the result the "strain". We divide the force by the cross-sectional area and call the result the "stress". The ratio of stress to strain is defined to be the Young's modulus of the material, which is then treated as homogeneous for purposes of finite element modeling of a structure.

This approximation is adequate for many applications. However, it ignores the reality of how a load applied on the surface of a concrete body is transmitted internally. Concrete is a heterogeneous material. It consists of small rocks (aggregate) of length scale about $2 \mathrm{~cm}$ held together by a weaker material (cement or paste). Since the aggregate inclusions may be in contact with each other, or nearly so, the actual force distribution within the material follows a tortuous path through the aggregate particles and their points of contact. This results in a quantitative and qualitative disparity between the local equations of solid mechanics theory using smoothed material properties and the way the material really behaves. Nonlocality in a random medium such as concrete has been treated in a number of references, for example [Willis 1985; Drugan and Willis 1996; Drugan 2003].

In the early 1980s, Bažant pioneered the application of nonlocal modeling to materials with damage. He demonstrated by a simple example that nonlocality is a necessary property of the elastic response in a material containing distributed defects [Bažant 1991]. In the same general spirit, the present paper

Sandia is a multiprogram laboratory operated by Sandia Corporation, a Lockheed Martin Company, for the United States Department of Energy's National Nuclear Security Administration under contract DE-AC04-94AL85000.

Keywords: composite, laminate, elasticity, nonlocality, peridynamics. 
derives nonlocal interactions that are implied by the use of a smoothed displacement field to model a heterogeneous microstructure. Unlike Bažant's analysis, the discussion here omits cracks and damage, and treats only the elastic response of the composite.

Nonlocality also arises in the study of plasticity, in which the finite sizes of dislocations and the distances between them interacts with the geometry of the system, as in the formation of geometrically necessary dislocations. Strain gradient models of plasticity, which incorporate a kind of weak nonlocality, have been developed to model such effects [Fleck et al. 1994; Bassani et al. 2000]. Much of the nonlocal literature concerns nonlocal operations on a damage variable, particularly its beneficial effects in reducing mesh dependence in numerical modeling [Bažant 1991; de Borst et al. 1995; Geers et al. 1999; Germain et al. 2007]. In a heterogeneous elastic material with a periodic microstructure, it has also long been recognized that nonlocal interactions may arise as a result of homogenization [Beran and McCoy 1970a; 1970b; Gambin and Kröner 1989; Boutin 1996; Bellieud and Bouchitté 1998; Fish et al. 2002; Cherednichenko et al. 2006; Chakraborty 2007]. Ben-Amoz [1975; 1976] and Ardiç, Santare, and Chou [Ardiç et al. 1989] incorporated aspects of nonlocality in models of composite material elasticity.

A large body of literature on nonlocal elasticity, much of which is highly mathematical, has been developed over the past five decades. In spite of this, nonlocal models are generally not adopted in the computational and analytical methods that engineers commonly use for applications. A typical analyst simply does not see why there should be nonlocal forces in a material at any scale above the molecular. Casual observers may conclude that, in spite of any benefits in regularizing finite element simulations, and regardless of compelling evidence from mathematical proofs, nonlocal models are not justified physically.

What apparently has been lacking in the nonlocal literature is a convincing mechanical picture of nonlocal interactions. In the present work, we consider how nonlocality arises in a specific, relatively simple heterogeneous system, based only on simple mechanical concepts. The micromechanical model uses only the standard equations of solid mechanics, yet it is shown that nonlocality appears in the global model derived from it. It is demonstrated that nonlocality arises from the decision to model the composite in terms of a smoothed displacement field, rather than arising from direct physical interactions across a finite distance. The nonlocal interaction forces can be included in the displacement equations of motion in various nonlocal theories. This example also permits us to compute how mesoscale geometrical dimensions combine with material properties to determine the length scale that applies in the nonlocal model.

\section{Microscale model of a composite}

Consider a composite material composed of alternating layers of stiff $(s)$ and compliant $(c)$ materials (Figure 1). The two constituent materials have the same density. The layers have thickness $2 h_{s}$ and $2 h_{c}$. Let $E_{s}$ and $E_{c}$ denote the Young's moduli and $\mu_{s}$ and $\mu_{c}$ the shear moduli of the materials. It is assumed that

$$
E_{s} \gg E_{c}, \quad \mu_{s} \gg \mu_{c} .
$$

Only the displacements $u_{s}$ and $u_{c}$ in the $x$-direction, that is, parallel to the layers, appear in the following approximate analysis.

The following analysis is based on a "shear-lag" model of the transfer of forces between the materials [Nairn 1997]. It is assumed that because of the disparity in elastic constants, $u_{s}$ is independent of $y$. The 


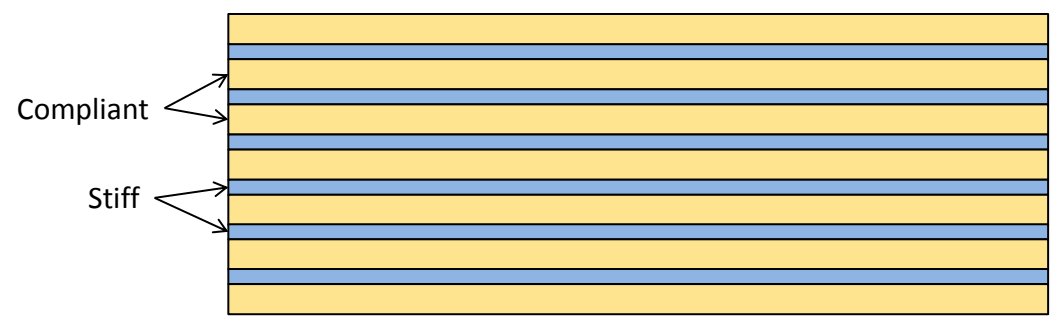

Figure 1. Composite composed of alternating stiff and compliant layers.

following ansatz is adopted: in the compliant material, a displacement field of the form

$$
u_{c}(x, y)=u_{s}(x)+\left(h_{c}^{2}-y^{2}\right) w(x)
$$

is assumed, where $w$ is a continuous function and $y=0$ is located at the midplane of a typical compliant layer (Figure 2). The shear traction on either surface of any compliant layer is found from

$$
\tau(x)=\mu_{c} \frac{\partial u_{c}}{\partial y}\left(x,-h_{c}\right)=2 \mu_{c} h_{c} w(x) .
$$

In the absence of body forces, a force balance on the cross-section of a stiff layer yields

$$
h_{s} \sigma_{s}^{\prime}(x)+\tau(x)=0,
$$

where $\sigma_{s}$ is the normal stress and a prime denotes $d / d x$. Using (2-2), (2-3), and the Hooke's law expression

$$
\sigma_{s}=E_{s} u_{s}^{\prime}
$$

the force balance on the stiff layers may be rewritten as

$$
E_{s} h_{s} u_{s}^{\prime \prime}(x)+2 \mu_{c} h_{c} w(x)=0 .
$$

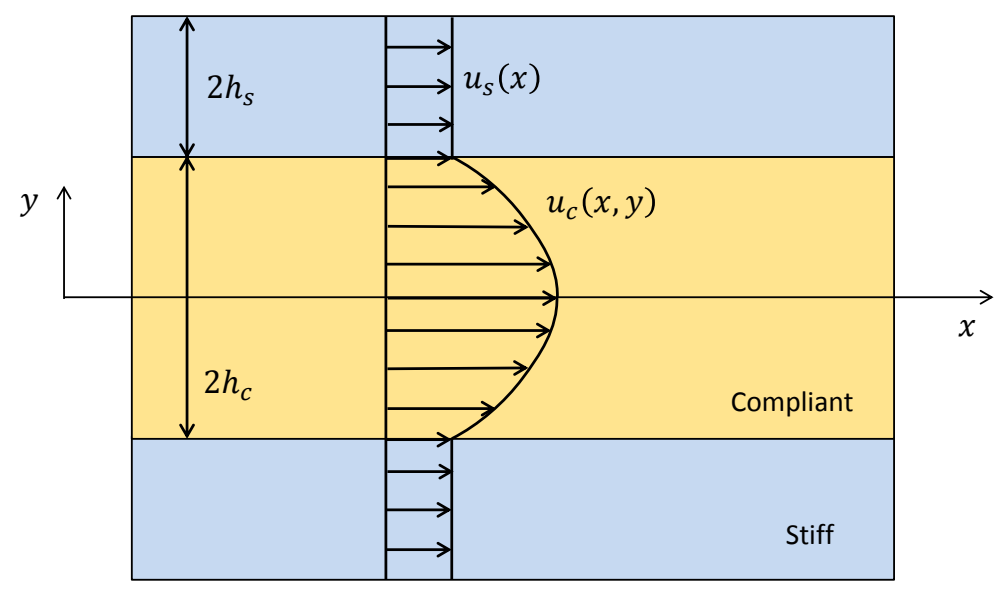

Figure 2. Axial displacement fields in the composite layers. 


\section{Equilibrium of a smoothed displacement field}

Define the smoothed (homogenized) displacement field to be the average at any $x$ over a cross-section through the layers:

$$
\bar{u}(x)=\frac{1}{h_{s}+h_{c}}\left[h_{s} u_{s}(x)+\int_{0}^{h_{c}} u_{c}(x, y) d y\right] .
$$

Using (2-1), this evaluates to

$$
\bar{u}(x)=u_{s}(x)+\alpha w(x),
$$

where

$$
\alpha=\frac{2 h_{c}^{3}}{3\left(h_{s}+h_{c}\right)} .
$$

Using (3-1) and (3-2), we remove $u_{s}$ from the force balance (2-4) and obtain

$$
\bar{u}^{\prime \prime}(x)=\alpha w^{\prime \prime}(x)-\frac{2 \mu_{c} h_{c}}{E_{s} h_{s}} w(x) .
$$

Our immediate objective is to analyze the forces in the composite for a prescribed $\bar{u}$ function. If $\bar{u}$ is prescribed, then (3-3) is a nonhomogeneous second-order linear ordinary differential equation (ODE) for $w$. The homogeneous part of the solution to this ODE (that is, for $\bar{u}$ either constant or a linear function of $x$ ) is

$$
w_{h}(x)=A e^{\lambda x}+B e^{-\lambda x},
$$

where

$$
\lambda=\sqrt{\frac{2 \mu_{c} h_{c}}{\alpha E_{s} h_{s}}}=\sqrt{\frac{3 \mu_{c}\left(h_{s}+h_{c}\right)}{E_{s} h_{s} h_{c}^{2}}},
$$

and where $A$ and $B$ are arbitrary constants.

Of particular interest is the choice of prescribed $\bar{u}^{\prime \prime}$ given by

$$
\bar{u}^{\prime \prime}(x)=\Delta(x),
$$

where $\Delta$ is the Dirac delta function. Kinematically, this choice means that the homogenized strain field $\bar{u}^{\prime}$ is given by the Heaviside step function

$$
\bar{u}^{\prime}(x)=H(x)
$$

(see Figure 3). For this choice, the solution, denoted $-G$, to the ODE (3-3) is

$$
w(x)=-G(x), \quad G(x)=k e^{-\lambda|x|},
$$

where

$$
k=\frac{1}{2 \alpha \lambda} .
$$

The easiest way to confirm that (3-6) and (3-7) provide a solution to (3-3) and (3-5) is to observe that by integrating (3-3) from $0^{-}$to $0^{+}$, the jump in $\bar{u}^{\prime}$ at the origin is given by

$$
\left[\bar{u}^{\prime}\right]=\alpha\left[w^{\prime}\right]-\frac{2 \mu_{c} h_{c}}{E_{s} h_{s}} \int_{0^{-}}^{0^{+}} w(x) d x .
$$




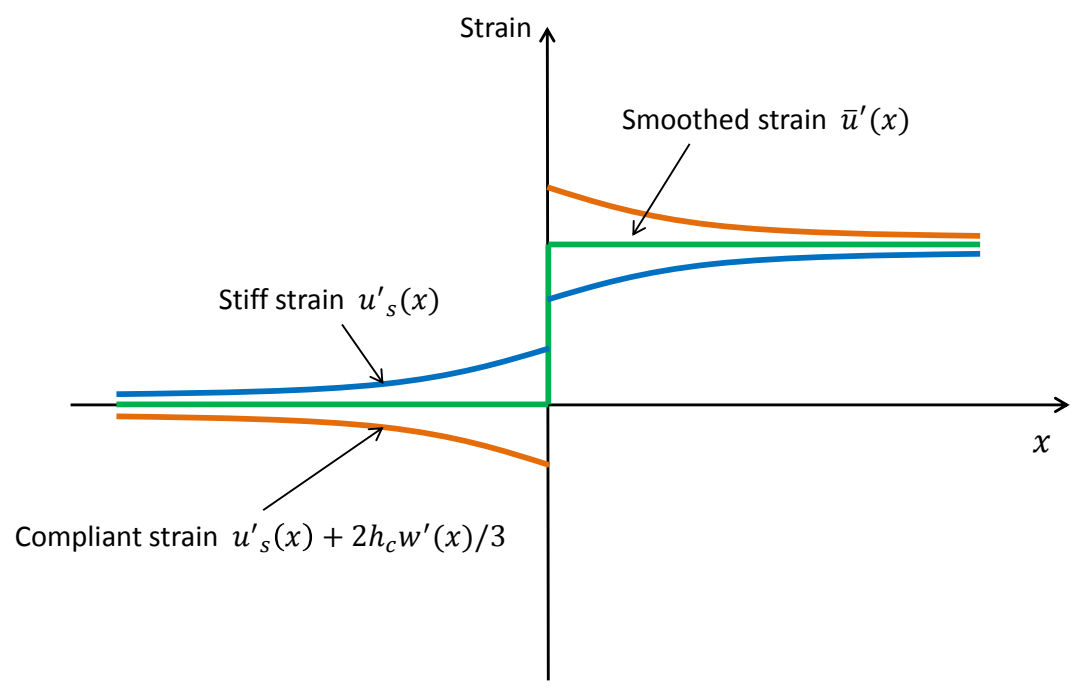

Figure 3. Strain fields in the stiff and compliant layers resulting from a prescribed smoothed displacement field in which there is a jump in strain at $x=0$.

By (3-6), $w$ is bounded (as well as continuous) everywhere, so the last term vanishes. Differentiation of $w$ yields

$$
w^{\prime}(x)=k \lambda(2 H(x)-1) e^{-\lambda|x|}
$$

Evidently this implies $\left[w^{\prime}\right]=2 k \lambda$. So, using (3-7), the jump condition (3-8) is satisfied.

Because the ODE (3-3) is linear, and because of the properties of the Dirac delta function, for an arbitrary prescribed function $\bar{u},(3-6)$ implies

$$
w(x)=-\int_{-\infty}^{\infty} \bar{u}^{\prime \prime}(p) G(x-p) d p .
$$

Next we compute the homogenized normal stress $\bar{\sigma}$ in the composite. From a force balance on a crosssection through the point $x$, this stress is found to be

$$
\bar{\sigma}(x)=\frac{1}{h_{s}+h_{c}}\left[h_{s} \sigma_{s}(x)+\int_{0}^{h_{c}} \sigma_{c}(x, y) d y\right],
$$

with

$$
\sigma_{s}(x)=E_{s} u_{s}^{\prime}(x), \quad \sigma_{c}(x)=E_{c} u_{c}^{\prime}(x) .
$$

Carrying out the integration in (3-10) using (2-1) and (3-1) leads to

$$
\bar{\sigma}(x)=\bar{E} \bar{u}^{\prime}(x)-\gamma w^{\prime}(x),
$$


where

$$
\begin{gathered}
\bar{E}=\frac{E_{s} h_{s}+E_{c} h_{c}}{h_{s}+h_{c}}, \\
\gamma=\alpha \bar{E}-\frac{2 E_{c} h_{c}^{3}}{3\left(h_{s}+h_{c}\right)}=\frac{2 h_{s} h_{c}^{3}\left(E_{s}-E_{c}\right)}{3\left(h_{s}+h_{c}\right)^{2}} .
\end{gathered}
$$

Rewriting (3-11) using (3-9) and (3-13) leads to

$$
\bar{\sigma}(x)=\bar{E} \bar{u}^{\prime}(x)+\gamma \int_{-\infty}^{\infty} \bar{u}^{\prime \prime}(p) G^{\prime}(x-p) d p .
$$

Note that $w$ no longer appears explicitly in this expression for the homogenized stress. The nonlocality inherent in modeling heterogeneous materials using a smoothed displacement field is now evident: values of $\bar{u}$ remote from $x$ contribute to the stress at $x$. This is a type of strong nonlocality. A more suggestive version of (3-14) may be obtained by integrating the last term by parts:

$$
\bar{\sigma}(x)=\bar{E} \bar{u}^{\prime}(x)+\gamma \int_{-\infty}^{\infty} \bar{u}^{\prime}(p) G^{\prime \prime}(x-p) d p .
$$

Differentiating (3-6) twice yields

$$
\begin{aligned}
G^{\prime \prime}(x) & =k \lambda^{2} e^{-\lambda|x|}-2 k \lambda \Delta(x) \\
& =\lambda^{2} G(x)-2 k \lambda \Delta(x) .
\end{aligned}
$$

Substituting (3-17) into (3-15), using the properties of the Dirac delta function, and using (3-7), (3-12), and (3-13) to simplify the constants yields

$$
\bar{\sigma}(x)=E_{c} \bar{u}^{\prime}(x)+\gamma \lambda^{2} \int_{-\infty}^{\infty} \bar{u}^{\prime}(p) G(x-p) d p .
$$

This expression is similar to the nonlocal stress used in Eringen's nonlocal theory [Eringen and Edelen 1972], because it involves taking a weighted average of strain.

\section{Nonlocal equations of motion}

Next we investigate how the nonlocal forces in the homogenized model appear in nonlocal equations of motion. With this goal in mind, we evaluate the acceleration at $x$ at a given time $t$. From the linear momentum balance in the absence of body forces, using the assumption that $\rho_{s}=\rho_{c}$, our expression for the net normal stress (3-18) implies

$$
\rho \ddot{\bar{u}}(x)=\bar{\sigma}^{\prime}(x)=E_{c} \bar{u}^{\prime \prime}(x)+\gamma \lambda^{2} \int_{-\infty}^{\infty} \bar{u}^{\prime}(p) G^{\prime}(x-p) d p .
$$

Integrating the last term by parts,

$$
\rho \ddot{\bar{u}}(x)=E_{c} \bar{u}^{\prime \prime}(x)+\gamma \lambda^{2} \int_{-\infty}^{\infty} \bar{u}(p) G^{\prime \prime}(x-p) d p .
$$


Using (3-16) in the last term, and introducing a body force field $b$ according to d'Alembert's principle, yields

$$
\rho \ddot{\bar{u}}(x)=E_{c} \bar{u}^{\prime \prime}(x)+\gamma k \lambda^{4} \int_{-\infty}^{\infty}(\bar{u}(p)-\bar{u}(x)) e^{-\lambda|x-p|} d p+b(x) .
$$

In this form, the model is similar to the nonlocal theory proposed by DiPaola, Failla, and Zingales [2009]. This form, like that of Kröner [1967], retains both local and nonlocal terms.

The peridynamic model seeks to eliminate any reference to strain, because one of its goals is to avoid using spatial derivatives of the deformation, thus making the theory compatible with discontinuities in displacement. The general form of the peridynamic equation of motion in one dimension [Silling 2000; Silling and Lehoucq 2010] is given by

$$
\rho \ddot{u}(x)=\int_{\mathscr{H}_{\mathbf{x}}} f(p, x, t) d p+b(x),
$$

where $\mathscr{H}_{\mathbf{x}}$ is a neighborhood of $x$ called the family of $x$. The radius of $\mathscr{H}_{\mathbf{x}}$, which is called the horizon, can be either finite or infinite. The function $f$ is called the bond force density. It can depend quite generally on the deformation of the family through suitable material models, including the effects of nonlinearity and damage. The peridynamic model is nonlocal because the family has nonzero size.

In linearized peridynamics [Silling 2010], the equation of motion (4-2) can be approximated by the following expression, which is formally the same as in the nonlocal theories of Kunin [1982; 1983] and Rogula [1982]:

$$
\rho \ddot{u}(x)=\int_{\mathscr{H}_{\mathbf{x}}} C(x, p)(u(p)-u(x)) d p+b(x),
$$

where $C$ is a function called the micromodulus. The equation of motion (4-1) that was derived from the microstructural model of a composite has strong similarities to the linearized peridynamic expression (4-3). Both equations contain strongly nonlocal terms; in (4-1) the horizon is infinite, although a reasonable approximation would be to cut off the nonlocal interactions outside a distance $r$ where the weighting term $e^{-\lambda r}$ is sufficiently small.

A local term involving $u^{\prime \prime}$ does not appear in the peridynamic equation (4-3). However, with the goal of representing this term in (4-1), it can be approximated by short-range interactions by using the same sort of manipulations used above. Using integration by parts, the following identities hold:

$$
u^{\prime \prime}(x)=\int_{-\infty}^{\infty} u^{\prime \prime}(p) \Delta(x-p) d p=\int_{-\infty}^{\infty} u^{\prime}(p) \Delta^{\prime}(x-p) d p=\int_{-\infty}^{\infty} u(p) \Delta^{\prime \prime}(x-p) d p .
$$

Using the approximation

$$
\Delta(x) \approx \phi(x):=\frac{\tau e^{-\tau|x|}}{2},
$$

where $\tau$ is a large constant, we compute from (4-4)

$$
\begin{aligned}
u^{\prime \prime}(x) & =\int_{-\infty}^{\infty} u(p) \Delta^{\prime \prime}(x-p) d p \approx \int_{-\infty}^{\infty} u(p) \phi^{\prime \prime}(x-p) d p \\
& =\int_{-\infty}^{\infty} u(p)\left(\frac{\tau^{3} e^{-\tau|x-p|}}{2}-\tau^{2} \Delta(x-p)\right) d p=\frac{\tau^{3}}{2} \int_{-\infty}^{\infty}(u(p)-u(x)) e^{-\tau|x-p|} d p .
\end{aligned}
$$


Thus, our model for the smoothed composite displacement field, (4-1), can be approximated to any level of accuracy by choosing a sufficiently large $\tau$ in the following model:

$$
\rho \ddot{\bar{u}}(x)=\int_{\mathscr{H}_{\mathbf{x}}}\left(\frac{E_{c} \tau^{3}}{2} e^{-\tau|x-p|}+\gamma k \lambda^{4} e^{-\lambda|x-p|}\right)(\bar{u}(p)-\bar{u}(x)) d p+b(x) .
$$

This is the peridynamic model (4-3) with the choice of micromodulus function given by

$$
C(x, p)=\frac{E_{c} \tau^{3}}{2} e^{-\tau|x-p|}+\gamma k \lambda^{4} e^{-\lambda|x-p|} .
$$

The foregoing discussion illustrates many of the key properties in understanding the role of nonlocality in the modeling of continua:

- Nonlocality is introduced by making the modeling decision to describe the problem using a smoothed displacement field rather than the detailed microstructural fields.

- Although the underlying microstructural model (in terms of the $u_{s}$ and $u_{c}$ fields) is local, the resulting smoothed model (in terms of $\bar{u}$ ) is nonlocal.

- Expressions called bond force densities in the peridynamic model of the form

$$
C(x, p)(\bar{u}(p)-\bar{u}(x))
$$

do not necessarily represent a direct nonlocal physical interaction (such as electrostatic forces) between $p$ and $x$.

- The applicable length scale in the nonlocal term, $1 / \lambda$, depends not only on the geometrical length scales $\left(h_{s}\right.$ and $h_{c}$ ), but also on the constituent material properties $E_{s}$ and $\mu_{c}$. If $E_{s} \gg \mu_{c}$, then this length scale can greatly exceed the layer thicknesses. This result is consistent with the computations by Pipes and Pagano [1970] which show that edge effects on the stresses in plies can extend over distances that substantially exceed the ply thicknesses.

- A peridynamic model can contain multiple length scales; in the case of the composite model in (4-5) these are $1 / \lambda$ and $1 / \tau$.

- By considering a displacement field of the form

$$
\bar{u}(x)=\varepsilon_{0} x+\frac{\beta}{2} x^{2},
$$

where $\beta$ is a constant, and applying (4-1), one finds that the quadratic term leads to an acceleration at $x=0$ given by

$$
\ddot{\bar{u}}(0)=\frac{4 \beta \gamma k \lambda}{\rho} .
$$

Since this acceleration is positive whenever the strain gradient $\beta$ is positive, this result means that positive strain gradient tends to increase the force on $x$. This is suggestive of experimental results that show stresses in real materials increase as the strain gradient is increased [Fleck et al. 1994].

The analogous local model for the composite in terms of the smoothed displacement field is

$$
\rho \ddot{\bar{u}}(x)=\bar{E} \bar{u}^{\prime \prime}(x)+b(x),
$$


where $\bar{E}$ is given by (3-12). Comparing this with (4-1), evidently the local term $\bar{E} \bar{u}^{\prime \prime}$ is replaced by a different local term $E_{c} \bar{u}^{\prime \prime}$ plus a nonlocal term. It is possible that a more detailed microstructural model, for example, a submicrostructural model that considers the features within each of the layers such as individual fibers, would further resolve the local term in (4-1). Based on the patterns emerging in the above derivations, it is plausible that a hierarchy of $N$ such submodels could be derived, resulting in a micromodulus of the form

$$
C(x, p)=C_{1}\left(x, p ; \lambda_{1}\right)+C_{2}\left(x, p ; \lambda_{2}\right)+\cdots+C_{N}\left(x, p ; \lambda_{N}\right),
$$

where each $C_{i}$ represents interactions with length scale $1 / \lambda_{i}$. By inference from (4-5), such terms might have the form

$$
C_{i}\left(x, p ; \lambda_{i}\right)=a_{i} e^{\lambda_{i}|x-p|},
$$

where the $a_{i}$ are constants.

\section{Nonlocality at the macroscale}

As noted in the previous section, the operative length scale in a heterogeneous material system depends on the constituent material properties as well as the geometrical length scale. However, the macroscale geometry of a body also combines with material properties to provide additional length scales. For example, consider the classic problem of an anisotropic plate under tension containing an open hole of radius $r$ (Figure 4). In the local theory, the stress $\sigma_{y y}$ as a function of position $x$ along the midplane was derived analytically by Lekhnists̄kii [1968]:

$$
\sigma_{y y}=\frac{\sigma_{\infty}}{2}\left\{2+\left(\frac{r}{x}\right)^{2}+3\left(\frac{r}{x}\right)^{4}+(2-n)\left[5\left(\frac{r}{x}\right)^{6}-7\left(\frac{r}{x}\right)^{8}\right]\right\},
$$

where

$$
n=\sqrt{2\left(\frac{E_{1}}{E_{2}}-v_{12}\right)+\frac{E_{1}}{G_{12}}},
$$

where the subscripts 1 and 2 denote the loading direction and transverse direction, respectively. From this, the stress concentration at the edge of the hole is found to be

$$
K_{\pi / 2}=1+n .
$$

The parameter $n$ tends to increase in anisotropic materials, particularly those that have greater stiffness in the loading $(y)$ direction. In addition to changing the stress concentration at the edge, anisotropy also changes the rate at which the stress decays with distance from the edge. Denote by $r_{0}$ the radius at which the stress in the classical solution drops off to half of its value at the edge, that is,

$$
\frac{\sigma_{y y}\left(r_{0}\right)}{\sigma_{y y}(r)}=\frac{1}{2} .
$$

Typical values of $r_{0}$ determined from (5-1) are given in Table 1. (Similar decay distance parameters play an important role in certain laminate failure models that implicitly recognize nonlocality [Whitney and Nuismer 1974; Ko 1985] by including an explicit length scale.) 


\begin{tabular}{|lccc|}
\hline Material & $n$ & $K_{\pi / 2}$ & $r_{0} / r$ \\
Isotropic & 2 & 3 & 1.52 \\
Fabric ply & 4.16 & 5.16 & 1.13 \\
Unidirectional ply & 10 & 11 & 1.07 \\
\hline
\end{tabular}

Table 1. Stress concentration and decay distance near an open hole in a plate.

Thus, in a unidirectional ply, the stress decays to half its value at the edge over a distance of only $7 \%$ of the hole radius. If the hole radius is $r=2.5 \mathrm{~mm}$, then this distance is $r_{0}-r=0.175 \mathrm{~mm}$, which is on the order of a typical ply thickness.

To put this result in the context of our previous discussion of length scales and nonlocality in heterogeneous media, suppose we compute the length scale implied by (3-4) with $h_{c}=h_{s}=0.1 \mathrm{~mm}, \mu_{c}=4 \mathrm{GPa}$, and $E_{s}=150 \mathrm{GPa}$. The resulting length scale for nonlocal interactions due to transfer of shear load between the constituent materials is

$$
\frac{1}{\lambda}=\sqrt{\frac{E_{s} h_{s} h_{c}^{2}}{3 \mu_{c}\left(h_{s}+h_{c}\right)}}=0.25 \mathrm{~mm} .
$$

Comparing this with our stress concentration decay distance $(0.175 \mathrm{~mm})$, the two values are similar. From this similarity, evidently nonlocality in a homogenized model due to the exchange of forces between the materials could play an important role in modeling the problem.

Toubal, Karama, and Lorrain [Toubal et al. 2005] measured the stress in a fabric composite laminate near an open hole as a function of position along the midplane of the specimen. They used an electronic speckle pattern interferometry measurement technique. This technique provides noncontact strain data with a spatial resolution of about $0.5 \mathrm{~mm}$. Their measurements showed that the actual stress concentration is much lower than what is predicted by the analytical results from the local theory [Lekhnists̄kii 1968]. Does nonlocality explain this difference?

To investigate this possibility, the peridynamic computational model Emu [Silling and Askari 2005] was applied to try to reproduce the measured stress concentration reported in [Toubal et al. 2005]. The material model used in the peridynamic computations was similar to that used in [Xu et al. 2008]. In this material model, peridynamic bonds parallel to the fibers have much greater stiffness than bonds in any other direction.

In the experiment, the hole diameter was $5.0 \mathrm{~mm}$, and the specimen width was $25 \mathrm{~mm}$. The specimen contained six plies, all epoxy reinforced by carbon fabric, with a total thickness of $2.28 \mathrm{~mm}$. The ply laminate properties were $E_{1}=51 \mathrm{GPa}, E_{2}=50 \mathrm{GPa}, v_{12}=0.06$, and $G_{12}=3.24 \mathrm{GPa}$. Since all the plies were identical in the experiment, shear forces between the plies are not significantly involved in the problem. Therefore, for purposes of estimating the nonlocal interaction distance $1 / \lambda$, the applicable geometric length scale is the fabric tow width. In other words, each tow acts like one of the layers in the microstructural model developed in Section 2. This view is supported by the X-ray diffraction studies [Davies et al. 2008] which show that in the vicinity of an open hole, the fabric tows deform more or less uniformly across the width of each tow. Under this assumption, and setting $h_{c}=h_{s}=1.25 \mathrm{~mm}$, which is a typical value for tow width, one finds from (3-4) that $1 / \lambda=2.0 \mathrm{~mm}$. In the computational model, the mesh spacing was $0.32 \mathrm{~mm}$ and the peridynamic horizon was $2.0 \mathrm{~mm}$. 


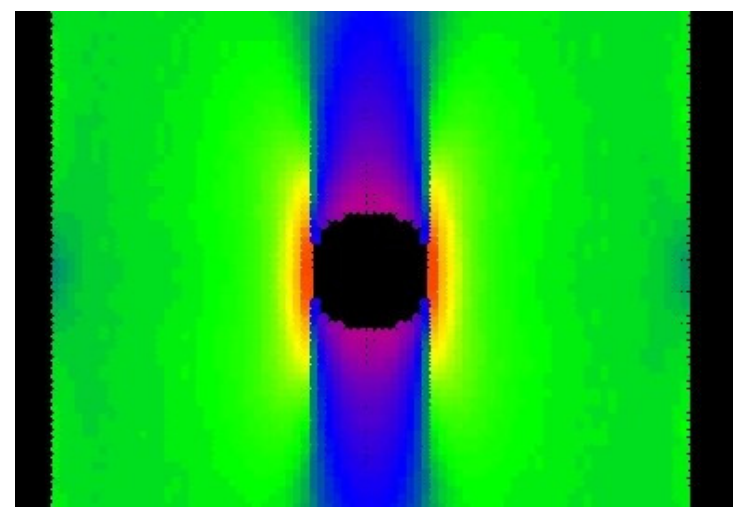

Figure 4. Stress contours in a simulated open-hole tension test in a composite. Note the large stress gradients.

As shown in Figure 4, the predicted contours of $\sigma_{y y}$ (the normal stress in the loading direction) show strong gradients in the vicinity of the hole. In fact, there are large gradients above and below the hole as well, due primarily to the relatively small shear modulus $G_{12}$, which is characteristic of fiber and fabricreinforced composites. In an isotropic material such as a typical metal, the contours of stress would be more diffuse. Figure 5 shows a comparison between the optically measured stress $\sigma_{y y}$ and the local theory of anisotropic media [Toubal et al. 2005]. The figure also shows the results from the peridynamic computational model. The results in this figure suggest that nonlocality helps improve the agreement

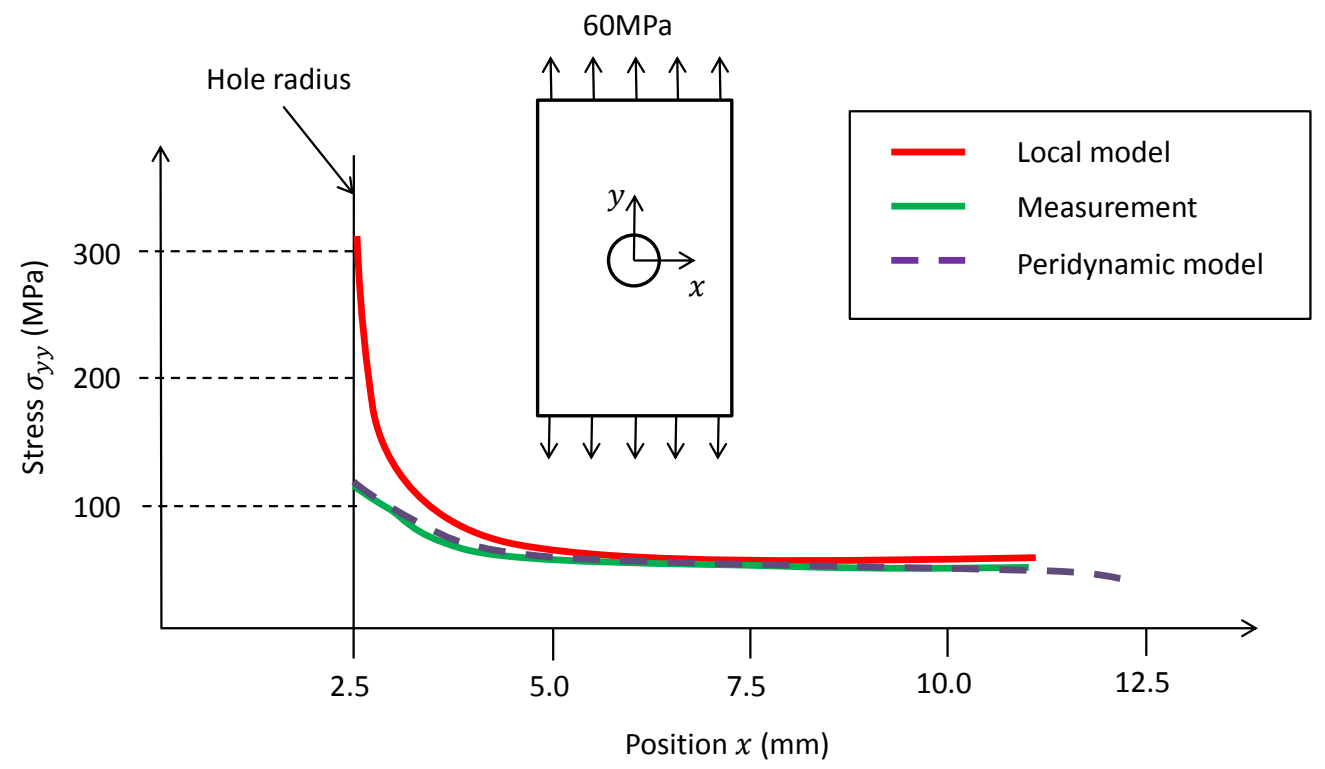

Figure 5. Comparison of stress along the midplane in an open-hole tension test on a fabric-reinforced composite. The local theory overpredicts the stress concentration compared with optically measured data [Toubal et al. 2005]. The peridynamic model offers improved agreement, apparently due to nonlocality. 
between a continuum model and measured data for stress concentrations in composites. This also helps explain why failure criteria in composites that rely solely on the predicted stress in the local theory tend to under-predict the failure load in open-hole tension and compression tests: these criteria are based on an over-prediction of the stress concentration [Soutis et al. 1991].

\section{Conclusions}

The purpose of this work is to show how a nonlocal model arises when we make the modeling decision to use a smoothed displacement field, rather than a detailed microstructural description. By considering the micromechanics of a layered composite under uniaxial stress, it was shown that nonuniformity of the displacement field across any cross-section leads to nonlocality in a homogenized model. The nonlocal effects appear only when the strain in the smoothed displacement field is nonconstant (that is, when a strain gradient is present). The nonlocal interactions, in this special case, can be represented using different nonlocal models, including those of Eringen and Kröner, as well as peridynamics. The peridynamic micromodulus function for the nonlocal interactions can be determined explicitly, although there is some arbitrariness in the kernel used to approximate the local term that appears in the peridynamic equation of motion. The length scale $1 / \lambda$ in the peridynamic model is determined not only by the microscale geometry of the composite, but also by the material properties of the constituent materials. In the peridynamic expression that was derived, interactions between material points separated by a finite distance necessarily occur, even though there are no direct physical interactions between these points in the microstructure.

In summary, nonlocality is not just a property of the physical system; it is also a property of the fields we choose to model the system with.

\section{References}

[Ardiç et al. 1989] E. S. Ardiç, M. H. Santare, and T.-W. Chou, "Stress fields in a composite material by means of a nonclassical approach", Int. J. Eng. Sci. 27:11 (1989), 1397-1405.

[Bassani et al. 2000] J. L. Bassani, A. Needleman, and E. van der Giessen, "Plastic flow in a composite: a comparison of nonlocal continuum and discrete dislocation predictions", Int. J. Solids Struct. 38:5 (2000), 833-853.

[Bažant 1991] Z. P. Bažant, "Why continuum damage is nonlocal: Micromechanics arguments", J. Eng. Mech. (ASCE) 117:5 (1991), 1070-1087.

[Bellieud and Bouchitté 1998] M. Bellieud and G. Bouchitté, "Homogenization of elliptic problems in a fiber reinforced structure: Nonlocal effects", Ann. Scuola Norm. Sup. Pisa Cl. Sci. (4) 26:3 (1998), 407-436.

[Ben-Amoz 1975] M. Ben-Amoz, "On wave propagation in laminated composites, I: Propagation parallel to the laminates", Int. J. Eng. Sci. 13 (1975), 43-56.

[Ben-Amoz 1976] M. Ben-Amoz, “A dynamic theory for composite materials”, Z. Angew. Math. Phys. 27 (1976), 83-99.

[Beran and McCoy 1970a] M. J. Beran and J. J. McCoy, "Mean field variations in a statistical sample of heterogeneous linearly elastic solids", Int. J. Solids Struct. 6 (1970), 1035-1054.

[Beran and McCoy 1970b] M. J. Beran and J. J. McCoy, "The use of strain gradient theory for analysis of random media", Int. J. Solids Struct. 6 (1970), 1267-1275.

[de Borst et al. 1995] R. de Borst, J. Pamin, R. H. J. Peerlings, and L. J. Sluys, "On gradient-enhanced damage and plasticity models for failure in quasi-brittle and frictional materials", Comput. Mech. 17:1-2 (1995), 130-141.

[Boutin 1996] C. Boutin, "Microstructural effects in elastic composites”, Int. J. Solids Struct. 33:7 (1996), 1023-1051. 
[Chakraborty 2007] A. Chakraborty, "Wave propagation in anisotropic media with non-local elasticity", Int. J. Solids Struct. 44:17 (2007), 5723-5741.

[Cherednichenko et al. 2006] K. D. Cherednichenko, V. P. Smyshlyaev, and V. V. Zhikov, "Non-local homogenized limits for composite media with highly anisotropic periodic fibres", Proc. Roy. Soc. Edinburgh Sect. A 136:1 (2006), 87-114.

[Davies et al. 2008] R. J. Davies, S. J. Eichhorn, J. A. Bennett, C. Riekel, and R. J. Young, "Analysis of the structure and deformation of a woven composite lamina using X-ray microdiffraction”, J. Mater. Sci. 43:20 (2008), 6724-6733.

[Di Paola et al. 2009] M. Di Paola, G. Failla, and M. Zingales, "Physically-based approach to the mechanics of strong non-local linear elasticity theory", J. Elasticity 97:2 (2009), 103-130.

[Drugan 2003] W. J. Drugan, "Two exact micromechanics-based nonlocal constitutive equations for random linear elastic composite materials", J. Mech. Phys. Solids 51:9 (2003), 1745-1772.

[Drugan and Willis 1996] W. J. Drugan and J. R. Willis, "A micromechanics-based nonlocal constitutive equation and estimates of representative volume element size for elastic composites", J. Mech. Phys. Solids 44:4 (1996), 497-524.

[Eringen and Edelen 1972] A. C. Eringen and D. G. B. Edelen, "On nonlocal elasticity”, Int. J. Eng. Sci. 10 (1972), $233-248$.

[Fish et al. 2002] J. Fish, W. Chen, and G. Nagai, "Non-local dispersive model for wave propagation in heterogeneous media: one-dimensional case", Int. J. Numer. Methods Eng. 54:3 (2002), 331-346.

[Fleck et al. 1994] N. A. Fleck, G. M. Muller, M. F. Ashby, and J. W. Hutchinson, "Strain gradient plasticity: Theory and experiment", Acta Metall. Mater. 42:2 (1994), 475-487.

[Gambin and Kröner 1989] B. Gambin and E. Kröner, "Higher-order terms in the homogenized stress-strain relation of periodic elastic media", Phys. Status Solidi B 151:2 (1989), 513-519.

[Geers et al. 1999] M. G. D. Geers, R. de Borst, and T. Peijs, "Mixed numerical-experimental identification of non-local characteristics of random-fibre-reinforced composites", Compos. Sci. Technol. 59:10 (1999), 1569-1578.

[Germain et al. 2007] N. Germain, J. Besson, and F. Feyel, "Composite layered materials: anisotropic nonlocal damage models", Comput. Methods Appl. Mech. Eng. 196:41-44 (2007), 4272-4282.

[Ko 1985] W. L. Ko, "Stress concentration around a small circular hole in the HiMAT composite plate", Technical Memorandum 86038, NASA, 1985.

[Kröner 1967] E. Kröner, "Elasticity theory of materials with long range cohesive forces", Int. J. Solids Struct. 3 (1967), 731742.

[Kunin 1982] I. A. Kunin, Elastic media with microstructure, I: One-dimensional models, Springer Series in Solid-State Sciences 26, Springer, Berlin, 1982.

[Kunin 1983] I. A. Kunin, Elastic media with microstructure, II: Three-dimensional models, Springer Series in Solid-State Sciences 44, Springer, Berlin, 1983.

[Lekhnists̄kii 1968] G. Lekhnistškii, Anisotropic plates, Gordon \& Breach, New York, 1968.

[Nairn 1997] J. A. Nairn, "On the use of shear-lag methods for analysis of stress transfer in unidirectional composites", $M e$ chanics of Materials 26:2 (1997), 63-80.

[Pipes and Pagano 1970] R. B. Pipes and N. J. Pagano, "Interlaminar stresses in composite laminates under uniform axial extension”, J. Compos. Mater. 4 (1970), 538-548.

[Rogula 1982] D. Rogula, Nonlocal theory of material media, CISM Courses and Lectures 268, Springer, Berlin, 1982.

[Silling 2000] S. A. Silling, "Reformulation of elasticity theory for discontinuities and long-range forces", J. Mech. Phys. Solids 48:1 (2000), 175-209.

[Silling 2010] S. A. Silling, "Linearized theory of peridynamic states", J. Elasticity 99:1 (2010), 85-111.

[Silling and Askari 2005] S. A. Silling and E. Askari, "A meshfree method based on the peridynamic model of solid mechanics", Comput. Struct. 83:17-18 (2005), 1526-1535.

[Silling and Lehoucq 2010] S. A. Silling and R. B. Lehoucq, "Peridynamic theory of solid mechanics", Adv. Appl. Mech. 44 (2010), 73-168.

[Soutis et al. 1991] C. Soutis, N. A. Fleck, and P. A. Smith, "Failure prediction technique for compression loaded carbon fibre-epoxy laminate with open holes", J. Compos. Mater. 25:11 (1991), 1476-1498. 
[Toubal et al. 2005] L. Toubal, M. Karama, and B. Lorrain, "Stress concentration in a circular hole in composite plate", Composite Structures 68:1 (2005), 31-36.

[Whitney and Nuismer 1974] J. M. Whitney and R. J. Nuismer, "Stress fracture criteria for laminated composites containing stress concentrations", J. Compos. Mater. 8:3 (1974), 253-265.

[Willis 1985] J. R. Willis, "The nonlocal influence of density variations in a composite”, Int. J. Solids Struct. 21 (1985), 805817.

[Xu et al. 2008] J. Xu, E. Askari, O. Weckner, and S. Silling, "Peridynamic analysis of impact damage in composite laminates", J. Aerosp. Eng. (ASCE) 21:3 (2008), 187-194.

Received 31 Jan 2014. Accepted 23 Feb 2014.

STEWART A. SILling: sasilli@sandia.gov

Multiscale Science Department, Sandia National Laboratories, P.O. Box 5800, MS-1322, Albuquerque, NM 87185-1322,

United States 


\title{
JOURNAL OF MECHANICS OF MATERIALS AND STRUCTURES
}

\author{
msp.org/jomms
}

\author{
Founded by Charles R. Steele and Marie-Louise Steele
}

EDITORIAL BOARD

ADAIR R. AGUIAR

KATIA BERTOLDI

University of São Paulo at São Carlos, Brazil

DAVIDE BIGONI

Harvard University, USA

IWONA JASIUK

University of Trento, Italy

Thomas J. PENCE

University of Illinois at Urbana-Champaign, USA

YASUHIDE SHINDO

Michigan State University, USA

DAVID STEIGMANN

Tohoku University, Japan

University of California at Berkeley

\section{ADVISORY BOARD}

J. P. CARTER University of Sydney, Australia

R. M. Christensen Stanford University, USA

G. M. L. GLADWELL University of Waterloo, Canada

D. H. Hodges Georgia Institute of Technology, USA

J. HUTCHINSON Harvard University, USA

C. HwU National Cheng Kung University, Taiwan

B. L. KARIHALOO University of Wales, UK

Y. Y. KIM Seoul National University, Republic of Korea

Z. Mroz Academy of Science, Poland

D. PAMPlonA Universidade Católica do Rio de Janeiro, Brazil

M. B. RubiN Technion, Haifa, Israel

A. N. SHUPIKOV Ukrainian Academy of Sciences, Ukraine

T. TARNAI University Budapest, Hungary

F. Y. M. WAN University of California, Irvine, USA

P. WRIGGERS Universität Hannover, Germany

W. YANG Tsinghua University, China

F. ZIEGLER Technische Universität Wien, Austria

PRODUCTION production@msp.org

SILVIO LEVY Scientific Editor

Cover photo: Mando Gomez, www.mandolux.com

See msp.org/jomms for submission guidelines.

JoMMS (ISSN 1559-3959) at Mathematical Sciences Publishers, 798 Evans Hall \#6840, c/o University of California, Berkeley, CA 94720-3840, is published in 10 issues a year. The subscription price for 2014 is US \$555/year for the electronic version, and $\$ 710 /$ year (+\$60, if shipping outside the US) for print and electronic. Subscriptions, requests for back issues, and changes of address should be sent to MSP.

JoMMS peer-review and production is managed by EditFLow ${ }^{\circledR}$ from Mathematical Sciences Publishers.

PUBLISHED BY

mathematical sciences publishers

nonprofit scientific publishing

http://msp.org/

(C) 2014 Mathematical Sciences Publishers 


\title{
Journal of Mechanics of Materials and Structures
}

\author{
Volume 9, No. $2 \quad$ March 2014
}

Plane waves at the boundary of two micropolar thermoelastic solids with distinct conductive and thermodynamic temperatures

Rajneesh Kumar, Mandeep Kaur and Satish C. RaJvanshi

Dynamic compression of square tube cellular structures RYAN L. HOLLOMAN, KaRTHIKEyan Kandan, VikRam DeshPande and HaYdn N. G. WadLey

Dynamic response of twin lined shells due to incident seismic waves

J. P. DWIVEdi, V. P. Singh and RADHA KRISHNA LAL 183

Solutions of the von Kármán plate equations by a Galerkin method, without inverting the tangent stiffiness matrix.

Honghua Dai, Xiaokuí Yue and Satya N.Atluri 195

Bimaterial lattices with anisotropic thermal expansion

Marina M. Toropova and Craig A. Steeves

Origin and effect of nonlocality in a composite STEWART A. Silling 